\title{
Low resolution scans can provide a sufficiently accurate, cost- and time-effective alternative to high resolution scans for 3D shape analyses
}

\author{
Ariel E Marcy ${ }^{\text {Corresp., }}{ }^{1}$, Carmelo Fruciano ${ }^{2}$, Matthew J Phillips ${ }^{3}$ ， Karine Mardon ${ }^{4,5}$, Vera Weisbecker ${ }^{1}$ \\ ${ }^{1}$ School of Biological Sciences, University of Queensland, Brisbane, Queensland, Australia \\ Institut de biologie de l'Ecole normale supérieure, Ecole normale supérieure, Université Paris, Paris, France \\ 3 School of Earth, Environmental and Biological Sciences, Queensland University of Technology, Brisbane, Queensland, Australia \\ 4 Centre for Advanced Imaging, University of Queensland, Brisbane, Queensland, Australia \\ 5 National Imaging Facility, University of Queensland, Brisbane, Queensland, Australia \\ Corresponding Author: Ariel E Marcy \\ Email address: a.marcy@uq.edu.au
}

Background. Advances in 3D shape capture technology have made powerful shape analyses, such as geometric morphometrics, more feasible. While the highly accurate micro-computed tomography $(\mu \mathrm{CT})$ scanners have been the "gold standard," recent improvements in 3D surface scanners may make this technology a faster, portable, and cost-effective alternative. Several studies have already compared the two devices but all use relatively large specimens such as human crania. Here we perform shape analyses on Australia's smallest rodent to test whether a 3D scanner produces similar results to a $\mu \mathrm{CT}$ scanner.

Methods. We captured 19 delicate mouse (Pseudomys delicatulus) crania with a $\mu$ CT scanner and a 3D scanner for geometric morphometrics. We ran multiple Procrustes ANOVAs to test how variation due to scan device compared to other sources such as biologically relevant variation and operator error. We quantified operator error as levels of variation and repeatability. Further, we tested if the two devices performed differently at classifying individuals based on sexual dimorphism. Finally, we inspected scatterplots of principal component analysis (PCA) scores for non-random patterns.

Results. In all Procrustes ANOVAs, regardless of factors included, differences between individuals contributed the most to total variation. The PCA plots reflect this in how the individuals are dispersed. Including only the symmetric component of shape increased the biological signal relative to variation due to device and due to error. 3D scans showed a higher level of operator error as evidenced by a greater spread of their replicates on the PCA, a higher level of multivariate variation, and a lower repeatability score. However, the 3D scan and $\mu C T$ scan datasets performed identically in classifying individuals based on intra-specific patterns of sexual dimorphism.

Discussion. Compared to $\mu \mathrm{CT}$ scans, we find that even low resolution 3D scans of very small specimens are sufficiently accurate to classify intra-specific differences. We also make 3 recommendations for best use of low resolution data. First, we recommend that extreme caution should be taken when analyzing the asymmetric component of shape variation. Second, using 3D scans generates more random error due to increased landmarking difficulty, therefore users should be conservative in landmark choice and avoid multiple operators. Third, using 3D scans introduces a source of systematic error relative to $\mu C T$ scans, therefore we recommend not combining them when possible, especially in studies expecting little biological variation. Our findings support increased use of low resolution 3D scans for most morphological 
studies; they are likely also applicable to low resolution scans of large specimens made in a medical CT scanner. As most vertebrates are relatively small, we anticipate our results will bolster more researchers in designing affordable large scale studies on small specimens with 3D surface scanners. 
1 Low resolution scans can provide a sufficiently accurate, cost- and time-effective

2 alternative to high resolution scans for 3D shape analyses

3

4 Authors: Ariel E. Marcy ${ }^{1}$, Carmelo Fruciano ${ }^{2}$, Matthew J. Phillips ${ }^{3}$, Karine Mardon ${ }^{4,5}$, Vera

$5 \quad$ Weisbecker $^{1}$

71 School of Biological Sciences, University of Queensland, Brisbane, Australia

82 Institut de biologie de l'Ecole normale supérieure (IBENS), Ecole normale supérieure,

9 CNRS, INSERM, PSL Université Paris, Paris, France

103 School of Earth, Environmental and Biological Sciences, Queensland University of

11 Technology, Brisbane, Australia

124 Centre for Advanced Imaging, University of Queensland, Brisbane, Australia

135 National Imaging Facility, University of Queensland, Brisbane, Australia

15 Corresponding Author:

16 Ariel E. Marcy

17 a.marcy@uq.edu.au 


\section{Abstract}

19 Background. Advances in 3D shape capture technology have made powerful shape analyses, such as geometric morphometrics, more feasible. While the highly accurate micro-computed

21 tomography $(\mu \mathrm{CT})$ scanners have been the "gold standard," recent improvements in 3D surface

22 scanners may make this technology a faster, portable, and cost-effective alternative. Several

23 studies have already compared the two devices but all use relatively large specimens such as

24 human crania. Here we perform shape analyses on Australia's smallest rodent to test whether a

$253 \mathrm{D}$ scanner produces similar results to a $\mu \mathrm{CT}$ scanner.

26 Methods. We captured 19 delicate mouse (Pseudomys delicatulus) crania with a $\mu \mathrm{CT}$ scanner

27 and a 3D scanner for geometric morphometrics. We ran multiple Procrustes ANOVAs to test

28 how variation due to scan device compared to other sources such as biologically relevant

29 variation and operator error. We quantified operator error as levels of variation and repeatability.

30 Further, we tested if the two devices performed differently at classifying individuals based on

31 sexual dimorphisms. Finally, we inspected scatterplots of principal component analysis (PCA)

32 scores for non-random patterns.

33 Results. In all Procrustes ANOVAs, regardless of factors included, differences between

34 individuals contributed the most to total variation. The PCA plots reflect this in how the

35 individuals are dispersed. Including only the symmetric component of shape increased the

36 biological signal relative to variation due to device and due to error. 3D scans showed a higher

37 level of operator error as evidenced by a greater spread of their replicates on the PCA, a higher

38 level of multivariate variation, and a lower repeatability score. However, the 3D scan and $\mu \mathrm{CT}$

39 scan datasets performed identically in classifying individuals based on intra-specific patterns of 40 sexual dimorphism. 
41 Discussion. Compared to $\mu \mathrm{CT}$ scans, we find that even low resolution 3D scans of very small

42 specimens are sufficiently accurate to classify intra-specific differences. We also make 3

43 recommendations for best use of low resolution data. First, we recommend that extreme caution

44 should be taken when analyzing the asymmetric component of shape variation. Second, using 3D

45 scans generates more random error due to increased landmarking difficulty, therefore users

46 should be conservative in landmark choice and avoid multiple operators. Third, using 3D scans

47 introduces a source of systematic error relative to $\mu \mathrm{CT}$ scans, therefore we recommend not

48 combing them when possible, especially in studies expecting little biological variation. Our

49 findings support increased use of low resolution 3D scans for most morphological studies; they

50 are likely also applicable to low resolution scans of large specimens made in a medical CT

51 scanner. As most vertebrates are relatively small, we anticipate our results will bolster more

52 researchers in designing affordable large scale studies on small specimens with 3D surface

53 scanners.

\section{Introduction}

56 An organism's shape reveals many facets of its biology, including its evolution, ecology, and

57 functional morphology. In the past three decades, geometric morphometrics has revolutionized

58 the field of shape research with better analysis and visualization of shape complexity (Rohlf \&

59 Marcus 1993; Zelditch et al. 2012). As imaging technology continues to advance, three-

60 dimensional (3D) data have become extremely common in geometric morphometric studies,

61 especially in the cases in which 2D data poorly represent the actual 3D object (Buser et al. 2017;

62 Cardini 2014; Fruciano 2016; Reig 1996). 3D capture methods include very high resolution yet

63 high cost micro-computed tomography $(\mu \mathrm{CT})$ scanners, which usually require time-intensive 
64 sectioning with specialized software. In contrast, 3D surface scanners offer lower acquisition

65 costs as well as faster scanning and processing, but has the disadvantage of generally lower

66 resolution, which limits its use on very small specimens (Fig. 1). For confident use of surface

67 scans in small specimens, it is therefore important to assess the measurement error introduced by 68 choosing a 3D surface scanner for geometric morphometrics.

Most vertebrates would be considered small, for example about two thirds of mammals are below 10kg (Weisbecker \& Goswami 2010), which would translate to small skeletal specimens.

72 Therefore, morphometric studies proposing large sample sizes must be very well funded to use a $\mu \mathrm{CT}$ scanner or have a low-cost option, such as a 3D surface scanner. Previous studies have compared $\mu \mathrm{CT}$ scans to 3D surface scans, however, these were all done in large animals, primarily primates (Badawi-Fayad \& Cabanis 2007; Fourie et al. 2011; Katz \& Friess 2014; Robinson \& Terhune 2017; Sholts et al. 2010; Slizewski et al. 2010). While these studies found low error and high repeatability in 3D surface scans similar to $\mu \mathrm{CT}$ scans, there was a suggestion that higher error occurred in the sample's smaller specimens (Badawi-Fayad \& Cabanis 2007;

79 Fourie et al. 2011). Other recent studies have conducted 3D geometric morphometric studies on small vertebrate skulls but nearly all have relied exclusively on $\mu \mathrm{CT}$ scanning (Cornette et al.

81 2013; Evin et al. 2011). The only exception we are aware of is (Muñoz-Muñoz et al. 2016), 82 which successfully used photogrammetry - a technique combining 2D photographs into a 3D 83 model - to analyze domestic mouse skulls, Mus musculus domesticus (Linnaeus, 1758).

84 Photogrammetry, like 3D surface scanning, is a low-cost alternative to $\mu \mathrm{CT}$ and comes with its own trade-offs in time and scan resolution (Katz \& Friess 2014). Compared to the new 86 generation of blue light surface scanners, photogrammetry requires more time for image 
87 acquisition and for file processing (Katz \& Friess 2014). A previous study on a single macaque

88 specimen reported inconsistent levels of error across operators and scanners, which contributed

89 to the lack of general pattern for differences across scanners/resolutions (Shearer et al. 2017).

90 However, using an interspecific dataset, (Fruciano et al. 2017) reported higher repeatability for

91 the higher resolution scans and $2.07-11.26 \%$ of total variance due to scan type (depending on

92 device, operator and landmark set combination). We expect that small specimens would

93 exacerbate any variation due to device and the interaction of device with other factors, such as

94 landmark choice and operator. More work comparing these different methods $-\mu \mathrm{CT}$ scanning,

95 3D surface scanning, and photogrammetry - will allow researchers to make an informed

96 decision. For example, for those with time constraints in museum collections, a fast 3D surface

97 scanner may be the best option if the resolution is suitable for specimen size.

99 The lower resolution of 3D surface scanners may increase both random and systematic

100 measurement error, which is exacerbated by small specimens because operators may have more

101 difficulty identifying landmark locations (Arnqvist \& Martensson 1998; Fruciano 2016).

102 Random error increases variance without changing the mean; this "noise" dilutes biologically

103 informative patterns and, in principle, decreases statistical power (Arnqvist \& Martensson 1998;

104 Fruciano 2016). By contrast, systematic error is non-randomly distributed, thus changing the

105 mean and introducing bias to the data (Arnqvist \& Martensson 1998; Fruciano 2016). Error

106 assessment can be done with repeated measures of the same individuals (e.g. Fruciano et al.

107 2017; Muñoz-Muñoz \& Perpiñán 2010; Robinson \& Terhune 2017) or by comparison to a "gold

108 standard" or ideal representation of the specimens (Fruciano 2016; Slizewski et al. 2010;

109 Williams \& Richtsmeier 2003). Repeated measure designs can uncover this systematic error, for 
110 example, if one 3D capture method differs from another in a specific, non-random, pattern

111 (Fruciano 2016; Fruciano et al. 2017). Furthermore, designs including repeated measures of the

112 same individuals allow partitioning of variance into components, quantifying error due to scan

113 device as compared to biologically-relevant sources of variation such as asymmetry (Fruciano

114 2016; Klingenberg et al. 2002; Klingenberg \& McIntyre 1998).

115

116 In this study, we quantify the error introduced by studying specimens of a size at the very lower

117 limits of commonly used portable surface scanners' resolution. This situation could also arise

118 when using relatively large specimens, which are nonetheless at the lower limit of a medical CT

119 scanner's resolution for example. We test whether the complex shape of very small specimens

120 can be adequately captured using an HDI109 3D surface scanner with a stated resolution of 80

$121 \mu \mathrm{m}$ as compared to a $\mu \mathrm{CT}$ scanner with a resolution of $28 \mu \mathrm{m}$. To do so, we use the delicate

122 mouse, Pseudomys delicatulus (Gould, 1842), one of the smallest rodents in the world with a 55-

$12375 \mathrm{~mm}$ head-and-body length (Breed \& Ford 2007). The miniscule P. delicatulus crania

$124(\sim 20 \mathrm{~mm})$ are at the edge of the HDI109 3D surface scanner's range thus providing an extreme

125 test of this scanning device (Fig. 1, Fig. 2). First, we tested whether variation due to scanning

126 device compared to other sources of variation (Fig. 2b). We also asked whether removing

127 asymmetric variation, a common practice in morphological studies when asymmetry is not of

128 interest, changed the results. Second, we tested whether the scanning devices differed in shape

129 variance and in operator error (as measured by repeatability) (Fig. 2c). We also explored how

130 including different types of landmarks impacted repeatability. Finally, we tested whether the

131 shape variation due to scanning device was large enough to impact a small study of intra-specific

132 shape variation using the biologically relevant signal of sexual dimorphism (Fig. 2d). 


\section{Methods}

\section{Data collection}

136 We selected 19 adult individuals, male and female, of Pseudomys delicatulus from the

137 Queensland Museum in Brisbane, Australia (specimen numbers and sexes in Additional File 1:

138 Table S1). The cranium from each individual was scanned at the Centre for Advanced Imaging at

139 the University of Queensland in a $\mu \mathrm{CT}$ scanner (Siemens Inveon PET/CT scanner). The scanner

140 was operated at $80 \mathrm{kV}$ energy, $250 \mu \mathrm{A}$ intensity with 540 projections per $360^{\circ}$, a medium-high

141 magnification with bin 2 was applied, and $2000 \mathrm{~ms}$ exposure time. The samples were scanned at

142 a nominal isotropic resolution of $28 \mu \mathrm{m}$. The data were reconstructed using a Feldkamp

143 conebeam back-projection algorithm provided by an Inveon Acquisition workstation from

144 Siemens (IAW version 2.1). Surface models were obtained using Mimics Research version 20.0.

146 Each cranium was also scanned by a HDI109 blue light surface scanner (LMI Technologies Inc.,

147 Vancouver, Canada) with a resolution of $80 \mu \mathrm{m}$. For brevity, we will refer to this method as 3D

148 scanning. For this method, the cranium was placed on a rotary table providing the scanner with

149360 views. To capture the entire shape, the cranium was scanned in three different orientations:

150 one ventral view with the cranium resting on the frontals and two dorsal views with the cranium

151 tipped to each side, resting on an incisor, auditory bulla, and zygomatic arch. To assist others in

152 replicating our HDI109 3D surface scanning on small specimens, we have included a Standard

153 Operating Procedure with our settings (Additional File 2: Supplementary Methods). 
155 After scanning every individual with both scan methods, we then replicated each 3D model three

156 times so that each individual was represented by 6 replicates, giving a total sample of 114 3D

157 models (Fig. 2a). Each 3D model was landmarked in Viewbox version 4.0 (dHAL software,

158 Kifissia, Greece; www.dhal.com; (Polychronis et al. 2013). To capture shape, we placed 58 fixed

159 landmarks, 145 semi-landmarks on curves, and 86 patch points (points that during sliding are

160 allowed to slide across a 3D surface defined by the 3D model and semi-landmark borders) for a

161 total of 289 points (Fig. 3, Additional File 3: Table S2). We used the template feature in

162 Viewbox to semi-automate the placement of semi-landmarks on curves and to fully automate the

163 placement of patch points. Our landmark design covered most important biological structures

164 except for the zygomatic arch (Fig. 3); we avoided this fine structure because dehydration and

165 loss of support from surrounding muscles during skeletonization almost certainly causes

166 specimen preparation error (Schmidt et al. 2010; Yezerinac et al. 1992).

167

168 Data analysis

169 The landmark coordinates for all 114 3D models were aligned using a generalized Procrustes

170 analysis followed by projection to the tangent space, as implemented in the $\mathrm{R}$

171 package geomorph (v. 3.0.5) (Adams 2016; Adams \& Otarola-Castillo 2013). Generalized

172 Procrustes analysis of each set of landmark coordinates removes differences in size, position, and

173 orientation, leaving only shape variation (Rohlf \& Slice 1990). Semi-landmarks and patches

174 were permitted to slide along their tangent directions to minimize Procrustes distance between

175 3D models (Gunz et al. 2005). The resulting Procrustes tangent coordinates were used as shape

176 variables in all subsequent shape analyses. All our statistical analyses were performed either in R 
177 (v. 3.3.3) using the R packages geomorph (v. 3.0.5) (Adams 2016; Adams \& Otarola-Castillo

178 2013) and Morpho (v. 2.5.1) (Schlager 2017) or using MorphoJ (v. 1.06d) (Klingenberg 2011).

180 First, asymmetry is a known source of variation within a sample (Klingenberg et al. 2002), so we

181 tested for it with MorphoJ's Procrustes ANOVA function and subsequently removed it (Fig. 2b).

182 Isolating the symmetric component of shape has been undertaken in other 3D surface scanner

183 studies where operator and device error have been of the same magnitude as asymmetric error

184 (Fruciano et al. 2017). Variation due to asymmetry is more impacted by operator error because

185 of its smaller effect sizes compared to variation among individuals (Fruciano 2016; Fruciano et

186 al. 2017; Klingenberg et al. 2010; Leamy \& Klingenberg 2005). This suggests that low

187 resolution studies on asymmetry would be negatively impacted. For this reason, we performed

188 most subsequent analyses on the symmetric shape component, with a few exceptions performed

189 for comparison. We then performed a PCA on the symmetric shape variables to visualize the

190 variation between individuals, within scan method replicates, and between scan method

191 replicates. As an exploratory analysis, PCA can help intuitively visualize both random error

192 (greater spread of one scan method replicate compared to the other) and systematic error

193 (repeated pattern of one scan method shifting relative to another). However, further analyses are

194 necessary to quantify these sources of error.

195

196 Second, our replicate design allowed us to assess whether an operator digitizing scans from one

197 device was more variable in landmark placement than when digitizing scans from the other

198 device (Fig. 2c). We did so by computing the Procrustes variance for each individual/device

199 combination. In geomorph, Procrustes variances are calculated for each set of observations (i.e. 
200 replicates) as the sum of the diagonal elements of the set's covariance matrix divided by the

201 number of observations (Adams 2016; Zelditch et al. 2012). We computed Procrustes variance

202 for each combination of individual and device so that Procrustes variance reflected only variation

203 due to digitization. We then compared Procrustes variance between devices using a box plot and

204 the permutational procedure implemented in geomorph. Next we quantified digitization

205 consistency by computing repeatability for each device using the analogue of the intraclass

206 correlation coefficient computed with the Procrustes ANOVA mean squares, as suggested by

207 (Fruciano 2016). This value is normally between 0 and 1 , with values close to 1 indicating much

208 larger variation due to the factor used in computing the Procrustes ANOVA (in our case,

209 variation among individuals) compared to residual variation (in our case, variation among

210 digitizations). In other words, comparing repeatability between devices gives similar information

211 to that obtained by the box plots of Procrustes variance but on a more easily interpretable scale

212 from 0 to 1 . We repeated our computations of repeatability for subsets of the data to test whether

213 introducing semi-landmarks on curves and surfaces (patch points) changed the repeatability

214 relative to a fixed landmark-only dataset. We did so for both 3D and $\mu \mathrm{CT}$ datasets to see if these

215 trends differed by scan device.

216

217 Finally, we investigated whether there is a difference between devices in a common task: the

218 correct classification of sexual dimorphism (Fig. 2d). We began with a Procrustes ANOVA in R

219 on the symmetric component for the subset of individuals with sex information $(\mathrm{n}=11$ distinct

220 individuals; $\mathrm{n}=663 \mathrm{D}$ models). This allowed us to gauge the magnitude of the effect of sexual

221 dimorphism compared to other sources of variation, including variation due to scan device. Then

222 with Morpho, we averaged the shape of each replicate triad for each device, and performed a 
223 between-group PCA using sex as group (Boulesteix 2005). Between-group principal component

224 analysis is an ordination technique which is gaining popularity in geometric morphometrics (eg.

225 Firmat et al. 2012; Franchini et al. 2016; Franchini et al. 2014; Fruciano et al. 2016; Fruciano et

226 al. 2014; Mitteroecker \& Bookstein 2011; Raffini et al. 2018; Schmieder et al. 2015; Seetah et al.

227 2012) However, it can be also thought of as a classification tool, as in the Morpho

228 implementation which allows performing leave-one-out cross-validation. We, then used cross-

229 validated classification accuracy as a measure of performance in classifying individuals based on 230 their sex.

231

232

Results

233 Analyses of shape variation

234 Our Procrustes ANOVA results indicate that variation among individuals $(\% \operatorname{Var}=48.3)$

235 contributes the most to total variance, with asymmetry (directional and fluctuating), device, and

236 operator error contributing the remainder (Table 1a). The \%Var values indicate that directional

237 asymmetry contributes a similar amount of variation as other sources of non-biological variation

238 and that fluctuating asymmetry accounts for much less than digitization error and variation

239 between devices (Table 1a). This means that using analyses of asymmetry with a combination of

$240 \mu \mathrm{CT}$ and 3D surface scans would likely be unreliable in specimens the size of delicate mice or

241 for specimens scanned at a similarly low resolution. The Procrustes ANOVA results for just the

242 3D data, confirms this observation in which digitization error is large compared to the

243 components of asymmetric variation (Table 1b). For the 3D dataset, the error term (Res/Rep)

244 contributes $17.8 \%$ of variation while asymmetry (Side) contributes $19.2 \%$. In other words, for

245 our 3D scan dataset, error contributes almost as much variation as asymmetry (Table 1b). The 
246 Procrustes ANOVA for just the $\mu \mathrm{CT}$ dataset, however, did not have this problem to the same

247 degree. Here, the error term (Res/Rep) contributes only $8.52 \%$ of variation while asymmetry

248 (Side) contributes 20.8\% (Table 1c). In other words, error contributes less than one half of the

249 contribution of asymmetry in the $\mu \mathrm{CT}$ dataset.

250

251 The Procrustes ANOVA on just the symmetric component of shape reports the individual shape

252 variation, representing biological variation, is $73.4 \%$ (Table 2). Differences between scan

253 devices represent $14.6 \%$ and the residuals encompassing differences among replicates or

254 operator error represent $12.0 \%$ of total variance (Table 2). Thus, our Procrustes ANOVA on the

255 symmetric component shows that most of the variation is due to biological sources but the

256 significance of the variation due to device may indicate systematic error.

258 The PCA on the symmetric component revealed that the first three principal components (PCs) account for $47.0 \%$ of total variation $(\mathrm{PC} 1=26.4 \%, \mathrm{PC} 2=12.0 \%, \mathrm{PC} 3=8.81 \%, \mathrm{n}=114)($ Fig.

4). Each of the remaining PCs accounted for $6 \%$ or less of total variation therefore we only considered the first three for the exploration of patterns of variation. Positive values along PC1 correspond to a larger braincase relative to the rostrum (Fig. 5a). Positive values along PC2 correspond to a wider frontal bone (Fig. 5b). Finally, positive values along PC3 correspond to a 264 more convex, dorsally-curved ventral surface (Fig. 5c).

The plot of the scores on PC1 and PC2 supports the results from the Procrustes ANOVA on the

267 symmetric component of shape in that most of the visible variation is between individuals, i.e.

268 clusters of each individual's replicates (Fig. 4a). Indeed, regardless of scanning device, replicates 
269 from the same individual cluster together (Fig. 4a). For most individuals, replicates occupy non-

270 overlapping regions of the plot except for those around the crowded mean shape near the origin

271 (Fig. 4a). Within each individual's variation on PCA scores, $\mu \mathrm{CT}$ replicates usually form a

272 tighter cluster than the $3 \mathrm{D}$ replicates (Fig. 4a). This pattern suggests that using $\mu \mathrm{CT}$ scans

273 introduces less random error than using 3D scans. Furthermore, within an individual, 3D scan

274 replicates tend to cluster closer to other 3D replicates while $\mu \mathrm{CT}$ scan replicates tend to cluster

275 closer to other $\mu \mathrm{CT}$ replicates (Fig. 4a). Indeed, for most individuals, 3D scan replicates score

276 lower than the $\mu \mathrm{CT}$ scan replicates from the same individual on both PC1 and PC2. These results

277 suggest the systematic error may be driven by $\mu \mathrm{CT}$ scans overestimating both braincase volume

278 and frontal bone width relative to 3D scans (Fig. 4a, Fig. 5a,b).

279

280 Overall, the scores along the first two PCs complement and provide an intuitive visualization for

281 the patterns of higher error in 3D scans and of systematic error between the scan devices as

282 observed in the Procrustes ANOVAs (Tables 1 and 2). The scores along PC1 and PC3 highlight

283 another possible systematic difference between 3D and $\mu \mathrm{CT}$ scans (Fig. 4b). The PC3 axis

284 displaces $\mu \mathrm{CT}$ replicates from 3D replicates such that variation in PC3 scores within individuals

285 is often larger than variation in PC3 scores among individuals (Fig. 4b). On the PC3 axis, almost

286 all 3D scan replicates had higher scores, which correspond to a more dorsally curved ventral

287 surface relative to their corresponding $\mu \mathrm{CT}$ scan replicates (Fig. 4b, Fig. 5c).

289 Procrustes variance and repeatability

290 To compare the digitization error in each scanning device dataset, we calculated the Procrustes

291 variance among the replicate triads of each individual. We found that Procrustes variance is 
292 significantly $(\mathrm{p}<0.001)$ higher in $3 \mathrm{D}$ scans $\left(\right.$ mean $\left.=1.31 \times 10^{-4}\right)$ than in $\mu \mathrm{CT}$ scans $($ mean $=$

$2934.76 \times 10^{-5}$ ) (Fig. 6). This means that digitizations are more variable in 3D scans than in $\mu \mathrm{CT}$

294 which is consistent with decreased clustering in 3D scans relative to $\mu \mathrm{CT}$ scans in the PCAs (Fig. $2954)$.

296

297 The repeatability for each scan dataset mirrored the Procrustes variance results. We found that 298 the $\mu \mathrm{CT}$ scan dataset had a repeatability of 0.896 and the 3D scan data had a repeatability of 2990.750 (Table 3a,d). This means operators are more successful at repeating their digitizations (i.e., 300 landmark placements) with $\mu \mathrm{CT}$ scans than with 3D scans.

301

302 To test how different types of landmarks impacted repeatability, we calculated repeatability for 303 combinations of landmark types for $3 \mathrm{D}$ and $\mu \mathrm{CT}$ datasets consisting of only the symmetric

304 component of shape (Table 3). Because sliding landmarks depend on the placement of fixed 305 landmarks (and patch points depend on both fixed and semi-landlandmark curves), we could not 306 isolate each type of landmark's repeatability. The analyses restricted to completely manually

307 placed fixed landmarks always had the lowest repeatability of the three types of landmarks

308 (Table 3c,f). Repeatability was always highest for the datasets including all three types of

309 landmarks including the semi-automated semi-landmarks and the completely automated patch

310 points (Table $3 \mathrm{a}, \mathrm{d})$. Higher repeatability in datasets with the sliding landmarks may result

311 because the sliding smooths out user placement error across replicates.

312

313 Analyses with a biological example: sexual dimorphism 
314 A small subset of our dataset had sex information $(\mathrm{n}=11 ; \mathrm{f}=7, \mathrm{~m}=4)$, allowing us to perform a

315 test of whether using different scan devices classify males and females according to shape with

316 the same level of accuracy. Our Procrustes ANOVA on the symmetric component of shape

317 variation using sex and device as factors found that shape differences due to device $(\mathrm{Rsq}=$

318 0.0646) and sex $(\mathrm{Rsq}=0.0952)$ are both significant $(\mathrm{p}<0.001)$. Both factors have relatively

319 small effect sizes, however, sex captures slightly more shape variation than device (Table 4).

320 However, the between-group PCAs do not suggest marked sexual dimorphism to begin with

321 (Fig. 7). Therefore, the subtlety of this biological signal could be the main reason for the small

322 contribution of sex to total variation. Finally, we performed a cross-validation test on the

323 between-group PCAs to assess which scan dataset can more reliably classify sexes based on

324 shape (Table 5). The results show that in this case, 3D scans and $\mu \mathrm{CT}$ scans perform identically 325 (overall classification accuracy $=63.6 \%$ ).

\section{Discussion}

In this study, we contrasted very high resolution $\mu \mathrm{CT}$ scans with their extreme opposite: $3 \mathrm{D}$ surface scans of very small specimens. Our low versus high resolution datasets allowed us to assess whether the low resolution scans still allow defensible investigations of biological shape variation. We found that despite the low quality of the 3D scans, sufficient amounts of biological variation are present to perform, at the very least, typical interspecific comparisons. In datasets with only very slight intra-specific differences, more difficulties in distinguishing biological

334 signal from the noise introduced by error during data collection. For example, the subtle sexual 335 dimorphism in our small sample was only just distinguished. However, we present three

336 considerations to make before using low resolution datasets. First, we found that variation due to 
337 scan device and digitizations is substantial relative to asymmetric variation. This makes low

338 resolution datasets a poor choice for studies on asymmetry. Second, using 3D scans creates more

339 random error due to increased landmarking difficulty, therefore care should be taken in landmark

340 choice, and possibly landmarking software and operator choice. Digitization error may also be

341 reduced by taking averages of repeated measurements (Arnqvist \& Martensson 1998; Fruciano

342 2016). Third, using 3D scans also introduces a source of systematic error relative to $\mu \mathrm{CT}$ scans,

343 therefore we recommend not combining them whenever possible (see also Fruciano et al. 2017),

344 and especially in studies on small intra-specific variation. In summary, with a few precautions

345 listed above, we expect that for studies with similarly sized skulls or similarly low resolution

346 scans, the variation due to error will be sufficiently low for successful detection of interspecific

347 shape differences.

348

349 Measurement error and 3D scan reliability

350 Systematic error between the two scan devices is shown by consistent displacement patterns in

351 the PCA. Indeed, across all three PC axes, the scans differ in how they measure concavity around

352 the braincase, frontal, and ventral surface. This systematic pattern could suggest that the 3D

353 scanner technology errs by adding volume to the digital specimen relative to the $\mu \mathrm{CT}$ scan but it

354 could also be the other way around with the $\mu \mathrm{CT}$ scan distorting the images to reduce volume.

355 Furthermore, even when using the symmetric component of shape, the percent of variation

356 contributed by scan device is quite substantial at about $14.5 \%$. Because scan device contributes

357 this much to variation and because systematic error between scan device exists, researchers

358 expecting very small variation due to biological sources would be advised not to combine 3D

359 scan and $\mu \mathrm{CT}$ scan datasets. 
361 While the two scan devices are usually comparable, using the low resolution 3D scans introduces

362 more digitization error than the higher resolution $\mu \mathrm{CT}$ scans, which likely reflects increased user

363 error due to lower resolution in 3D scans. This increased random error is reflected in both the

364 larger point clouds of 3D replicates relative to $\mu \mathrm{CT}$ replicates in the PCAs, the higher Procrustes

365 variance, and the lower repeatability score of 3D scans, particularly of manually-placed fixed

366 landmarks. As expected, we found that the low resolution 3D scans were more difficult to

367 landmark because key cranial features such as sutures and smaller processes were less distinct

368 (Fig. 1). Nevertheless, our overall 3D scan repeatability score of 0.75 with symmetric data

369 appears consistent with the literature: it is much lower than 3D scanned human-sized skulls -

370 above 0.95 (Badawi-Fayad \& Cabanis 2007; Fourie et al. 2011) but it is approaching the range of

371 3D scanned macropodoids (e.g., kangaroos) - 0.78-0.98, depending on device and landmark

372 choice (Fruciano et al. 2017). This trend of decreasing repeatability with decreasing body size

373 may reflect measurement error becoming a larger percentage of overall size (Robinson \&

374 Terhune 2017). Relatedly, recent work has shown that excluding a few unreliable landmarks, or

375 those with greater variability in placement, can significantly increase repeatability (Fruciano et

376 al. 2017). This may be especially true for small specimens, for which small variations from the

377 landmark location represent a larger percentage of their overall size.

378

379 Our repeatability tests on different combinations of landmark types suggest that fixed landmarks

380 suffer the most from decreased resolution and the associated increased user error while patch

381 points suffer the least. We interpret these results to mean that the (semi-) automatic placement of

382 semi-landmark curves and patches is more consistent in placing points compared to a human 
383 operator placing fixed landmarks, regardless of whether the automatic placement is "correct" or

384 not. It is important to note that while semi-landmarks were "semi-automated", the user still

385 manually defined the curve they slid along for each specimen. Furthermore, this curve is

386 bounded by user-placed fixed landmarks. Therefore, the increased repeatability with increasing

387 automation could also be due to the increased degrees of freedom afforded to landmarks during

388 sliding: fixed with zero degrees, semi-landmarks with one degree, and patch points with two.

389 The sliding, by removing variation tangential to a certain direction, will reduce the variance in

390 those points which will appear to vary less so it would be expected that these points will

391 contribute less overall variation when combined with the fixed landmarks.

392

393 This study did not look at multiple operator error which can be considerable, particularly if

394 difficult landmarks are included (Fruciano et al. 2017). If inter-operator error were combined

395 with the resolution-driven measurement error found here, it is possible that biological signal

396 would diminish to a degree that could not support even interspecific comparisons.

398 Measurement error introduced by scanning device compared to biological variation

399 The challenge of any quantitative measurement study is to minimize measurement error

400 introduced from various sources (in our case, device, resolution, and observer) relative to the

401 "true" signal of biological variation. For subtle sources of biological variation, such as

402 asymmetry, our results show that the error associated with collecting data from 3D scans

403 contributes the same amount of variation as asymmetry. Therefore, a low resolution study of

404 asymmetry with 3D scans would likely be unreliable unless appropriate arrangements were made

405 to reduce error (Fruciano 2016), whereas $\mu \mathrm{CT}$ scans may be more suitable for these types of 
406 studies. In the case of inter-observer error, which is another common source of measurement

407 error, several studies suggest that interspecific variation can overwhelm inter-observer error such

408 that this does not pose an issue with the correct interpretation of results (Robinson \& Terhune

409 2017).

410

411 In our test on the ability of different scan devices to classify according to sexual dimorphism, we

412 showed that while variation contributed by each source was similar (and that from scan device

413 slightly lower), both scan datasets presented a small sexually dimorphic pattern and supported

414 the same classification performance. This suggests that 3D scans may even be acceptable for

415 detecting some intra-specific patterns. However, this was a small sample $(\mathrm{n}=11)$ and further

416 studies with larger datasets would improve confidence for using 3D scans for intra-specific

417 studies. Studies based on larger datasets might also be able to better highlight differences in

418 classification performance between devices, if any. Nevertheless, it is promising that 3D scans

419 and $\mu \mathrm{CT}$ scans performed equally even at such a small sample size for such a subtle intra-

420 specific signal.

421

422 Choosing a digitization device: 3D surface scanning versus $\boldsymbol{\mu C T}$ versus photogrammetry

423 With many options for digitizing 3D specimens available, decisions on the acquisition mode

424 must consider price, scanning time, processing time, portability, and scan resolution. The one-off

425 investment of a relatively high resolution 3D surface scanner such as the HDI109 provided a

426 model portable enough to take on airplanes and with fast scanning and processing times. Our

427 model took 10 minutes from starting the scan to the finished surface file, but note that larger

428 specimens requiring multiple sub-scans will take longer. These fast acquisition times are an asset 
429 in collection efforts that rely on expensive and time-limited museum travel. For example, one of

430 us (AEM) digitized over 100 individuals in one week using the same scanning protocol.

431 However, the quality and speed of scanning varies by model; for example, other 3D surface

432 scanners could take over 45 minutes to capture one specimen and may also require more effort to 433 process scans (Katz \& Friess 2014).

435 Compared to 3D surface scanners, $\mu \mathrm{CT}$ scanners provide much higher resolution, which in this 436 study translated into less measurement error. However, uCT facilities are not widely accessible, 437 not mobile, and tend to be more expensive. Depending on the facility, $\mu \mathrm{CT}$ scanning involves 438 transport to the facility, scanning either by the operator, processing scans into image stacks, and

439 finally loading scans into specialized (and frequently high-cost) software to do the 3D

440 reconstruction. These reconstructions can be time consuming especially if the cranium needs to

441 be separated from the mandibles. Finally, specimens need to be loaned from their collections for

$442 \mu \mathrm{CT}$ acquisition, which requires specimen transport and curator permission and is particularly

443 difficult when large numbers of specimens from distant locations need to be scanned.

445 This study did not investigate photogrammetry, which is another and increasingly popular 446 method for digitizing 3D shape. This method uses software to align 2D photographs taken from 447 many different views into a 3D file. Photogrammetry is much cheaper and more portable than 3D 448 surface scanning since it only requires a camera of suitable resolution and very affordable photo449 alignment software like Agisoft PhotoScan (Agisoft LLC, St. Petersburg, Russia;

450 www.agisoft.com). The trade-offs are that in our experience, photogrammetry takes at least three 451 times longer to acquire the photos, it involves higher risk of human error or inconsistency during 
452 photography, and it requires an order of magnitude more time to align the photos into a 3D

453 digital file. While photo-alignment can be done at convenience after photography, the greater

454 time required to capture enough photos may be a deciding factor for researchers with time

455 limitations in museum collections. As for scan resolution, photogrammetry may perform better

456 than 3D surface scanners in some cases (Fourie et al. 2011) or at least provide an acceptable

457 alternative (Katz \& Friess 2014; Muñoz-Muñoz et al. 2016).

458

459 Scan resolution is not the only consideration when choosing a scan device as its unique

460 requirements for 3D model processing may increase image noise and therefore landmarking

461 difficulty. Compared to $\mu \mathrm{CT}$ scanning, 3D scans tend to be both noisier and require more model

462 processing before 3D model export. Specifically, artificial smoothing and hole-filling may

463 change the topography of the 3D mesh. Therefore, the comparison we have presented here is not

464 just a comparison of resolutions but also a comparison of 3D model generation. The methods we 465 provide in the supplementary files (Additional File 2: Supplementary Methods) represent the 466 settings we found to decrease noise, however, the software also required some model smoothing 467 and hole-filling before export. We recommend that researchers take these additional sources of 468 image modification into account during their landmark choice and study design.

\section{Conclusions}

471 Here, we have shown that a 3D surface scanner can provide an acceptable alternative to a $\mu \mathrm{CT}$

472 scanner for assessing biological signal of 3D shape even in small specimens that are at the limits

473 of 3D scanner resolution. Our analyses specifically showed that first, error contributes to a higher

474 percentage of variation in $3 \mathrm{D}$ scan datasets than in $\mu \mathrm{CT}$ scan datasets of the same small 
475 specimens. As a result, we conclude that 3D scans are usually not appropriate for studies on very

476 small sources of variation like fluctuating asymmetry. Second, we show that 3D scan datasets

477 have a lower repeatability of landmark placement, especially for fixed landmarks, as compared

478 to $\mu \mathrm{CT}$ scans. Relatedly, our comparisons of repeatability on data with asymmetry to the same

479 data without asymmetry - i.e. having bilateral symmetry - support analyzing the bilaterally

480 symmetrical data of landmarks from low resolution scans. Finally, we use a preliminary study of

481 sexual dimorphism to suggest that despite elevated error and shape variance, bilaterally

482 symmetrical datasets from 3D scans can support male versus female classification based on

483 small biological differences as well as $\mu \mathrm{CT}$ datasets can. In summary, while 3D scans are a

484 promising alternative, exploratory pilot studies of measurement error like this one are advisable

485 when practically possible (see also Fruciano 2016).

486

487 Furthermore, the best 3D capture method will vary based on the study's design, expected effect

488 size for the biological variation of interest, and the researcher's limitations on time, money, and

489 travel. In addition to image resolution requirements, it is wise to assess the time it will take to

490 capture and process each specimen as well as portability needs. We recommend a preliminary

491 test on multiple devices - including surface scanners - to determine how levels of error compare

492 to biological signal and whether there is substantial systematic error. Doing so may provide a

493 defensible alternative to an expensive and time consuming large-scale acquisition of $\mu \mathrm{CT}$ scans

494 including for studies on very small specimens.

495

496 Acknowledgements 
497 We would like to thank Cruise Speck for assistance with Viewbox software and Dr. Heather

498 Janetzki for hosting us in the mammal collections at the Queensland Museum.

499

500 Abbreviations

501 Landmark (LM)

502 Micro-computed tomography $(\mu \mathrm{CT})$

503 Principal component analysis (PCA)

504 Principal component (PC)

505 Three-dimensional (3D)

506

507 References

508 Adams D, ML Collyer, and E. Sherratt. 2016. geomorph: Software for geometric morphometric $509 \quad$ analyses. 3.0 ed.

510 Adams DC, and Otarola-Castillo E. 2013. geomorph: an r package for the collection and analysis

511 of geometric morphometric shape data. Methods in Ecology and Evolution 4:393-399.

$512 \quad 10.1111 / 2041-210 x .12035$

513 Arnqvist G, and Martensson T. 1998. Measurement error in geometric morphometrics: Empirical

514 strategies to assess and reduce its impact on measures of shape. Acta Zoologica

515 Academiae Scientiarum Hungaricae 44:73-96.

516 Badawi-Fayad J, and Cabanis EA. 2007. Three-dimensional procrustes analysis of modern

517 human craniofacial form. Anatomical Record-Advances in Integrative Anatomy and

518 Evolutionary Biology 290:268-276. 10.1002/ar.20442 
519 Boulesteix A-L. 2005. A note on between-group PCA. International Journal of Pure and $520 \quad$ Applied Mathematics 19:359-366.

521 Breed B, and Ford F. 2007. Native mice and rats: CSIRO Publishing.

522 Buser TJ, Sidlauskas BL, and Summers AP. 2017. 2D or Not 2D? Testing the Utility of 2D Vs. 523 3D Landmark Data in Geometric Morphometrics of the Sculpin Subfamily Oligocottinae (Pisces; Cottoidea). The Anatomical Record.

Cardini A. 2014. Missing the third dimension in geometric morphometrics: how to assess if 2D images really are a good proxy for 3D structures? Hystrix-Italian Journal of Mammalogy 25:73-81. 10.4404/hystrix-25.2-10993

Cornette R, Baylac M, Souter T, and Herrel A. 2013. Does shape co-variation between the skull and the mandible have functional consequences? A 3D approach for a 3D problem. Journal of Anatomy 223:329-336. 10.1111/joa.12086

531

532

Evin A, Horacek I, and Hulva P. 2011. Phenotypic diversification and island evolution of pipistrelle bats (Pipistrellus pipistrellus group) in the Mediterranean region inferred from geometric morphometrics and molecular phylogenetics. Journal of Biogeography 38:2091-2105. 10.1111/j.1365-2699.2011.02556.x

Firmat C, Schliewen UK, Losseau M, and Alibert P. 2012. Body shape differentiation at global and local geographic scales in the invasive cichlid Oreochromis mossambicus. Biological Journal of the Linnean Society 105:369-381. 10.1111/j.1095-8312.2011.01802.x

Fourie Z, Damstra J, Gerrits PO, and Ren YJ. 2011. Evaluation of anthropometric accuracy and reliability using different three-dimensional scanning systems. Forensic Science International 207:127-134. 10.1016/j.forsciint.2010.09.018 
541 Franchini P, Colangelo P, Meyer A, and Fruciano C. 2016. Chromosomal rearrangements,

542 phenotypic variation and modularity: a case study from a contact zone between house

543 mouse Robertsonian races in Central Italy. Ecology and Evolution 6:1353-1362.

544 10.1002/ece3.1912

545 Franchini P, Fruciano C, Spreitzer ML, Jones JC, Elmer KR, Henning F, and Meyer A. 2014. Genomic architecture of ecologically divergent body shape in a pair of sympatric crater

Fruciano C. 2016. Measurement error in geometric morphometrics. Development Genes and Evolution 226:139-158. 10.1007/s00427-016-0537-4

Fruciano C, Celik MA, Butler K, Dooley T, Weisbecker V, and Phillips MJ. 2017. Sharing is caring? Measurement error and the issues arising from combining 3D morphometric datasets. Ecology and Evolution 7:7034-7046. 10.1002/ece3.3256

Fruciano C, Franchini P, Raffini F, Fan S, and Meyer A. 2016. Are sympatrically speciating Midas cichlid fish special? Patterns of morphological and genetic variation in the closely related species Archocentrus centrarchus. Ecology and Evolution 6:4102-4114.

Fruciano C, Pappalardo AM, Tigano C, and Ferrito V. 2014. Phylogeographical relationships of Sicilian brown trout and the effects of genetic introgression on morphospace occupation. Biological Journal of the Linnean Society 112:387-398.

Gunz P, Mitteroecker P, and Bookstein FL. 2005. Semilandmarks in three dimensions. Modern Morphometrics in Physical Anthropology 73-98. 10.1007/0-387-27614-9_3

561 Katz D, and Friess M. 2014. 3D from standard digital photography of human crania-a preliminary assessment. American Journal of Physical Anthropology 154:152-158. 
563 Klingenberg C, Wetherill L, Rogers J, Moore E, Ward R, Autti-Rämö I, Fagerlund Å, Jacobson

564 S, Robinson L, and Hoyme H. 2010. Prenatal alcohol exposure alters the patterns of 565 facial asymmetry. Alcohol 44:649-657.

566 Klingenberg CP. 2011. MorphoJ: an integrated software package for geometric morphometrics. Molecular Ecology Resources 11:353-357. 10.1111/j.1755-0998.2010.02924.x

Klingenberg CP, Barluenga M, and Meyer A. 2002. Shape analysis of symmetric structures: Quantifying variation among individuals and asymmetry. Evolution 56:1909-1920.

Klingenberg CP, and McIntyre GS. 1998. Geometric morphometrics of developmental instability: Analyzing patterns of fluctuating asymmetry with procrustes methods. Evolution 52:1363-1375. 10.2307/2411306

573 574 575 576

Leamy LJ, and Klingenberg CP. 2005. The genetics and evolution of fluctuating asymmetry. Annual Review of Ecology, Evolution, and Systematics 36:1-21.

Mitteroecker P, and Bookstein F. 2011. Linear discrimination, ordination, and the visualization of selection gradients in modern morphometrics. Evolutionary Biology 38:100-114. $10.1007 / \mathrm{s} 11692-011-9109-8$

Muñoz-Muñoz F, and Perpiñán D. 2010. Measurement error in morphometric studies: comparison between manual and computerized methods. Annales Zoologici Fennici 47:46-56.

Muñoz-Muñoz F, Quinto-Sánchez M, and González-José R. 2016. Photogrammetry: a useful tool for three-dimensional morphometric analysis of small mammals. Journal of Zoological Systematics and Evolutionary Research. 
584 Polychronis G, Christou P, Mavragani M, and Halazonetis DJ. 2013. Geometric morphometric

585 3D shape analysis and covariation of human mandibular and maxillary first molars.

586 American Journal of Physical Anthropology 152:186-196. 10.1002/ajpa.22340

587

588

589

590

Raffini F, Fruciano C, and Meyer A. 2018. Morphological and genetic correlates in the left-right asymmetric scale-eating cichlid fish of Lake Tanganyika. Biological Journal of the Linnean Society 124:67-84.

Reig S. 1996. Correspondence between interlandmark distances and caliper measurements. Advances in Morphometrics 284:371-385.

Robinson C, and Terhune CE. 2017. Error in geometric morphometric data collection: Combining data from multiple sources. American Journal of Physical Anthropology 164:62-75.

Rohlf FJ, and Marcus LF. 1993. A revolution in morphometrics. Trends in Ecology \& Evolution 8:129-132.

Rohlf FJ, and Slice D. 1990. Extensions of the Procrustes method for the optimal superimposition of landmarks. Systematic Zoology 39:40-59. 10.2307/2992207

Schlager S. 2017. Morpho and Rvcg -- Shape Analysis in R. In: Guoyan Zheng SLaGS, ed. Statistical Shape and Deformation Analysis: Academic Press, 217--256.

Schmidt EJ, Parsons TE, Jamniczky HA, Gitelman J, Trpkov C, Boughner JC, Logan CC, Sensen CW, and Hallgrimsson B. 2010. Micro-computed tomography-based phenotypic approaches in embryology: procedural artifacts on assessments of embryonic craniofacial growth and development. BMC Developmental Biology 10. 10.1186/1471-213x-10-18 
605 Schmieder DA, Benitez HA, Borissov IM, and Fruciano C. 2015. Bat species comparisons based 606 on external morphology: a test of traditional versus geometric morphometric approaches.

607 PLOS One 10. 10.1371/journal.pone.0127043

608 Seetah TK, Cardini A, and Miracle PT. 2012. Can morphospace shed light on cave bear spatial609 temporal variation? Population dynamics of Ursus spelaeus from Romualdova pećina and Vindija, (Croatia). Journal of Archaeological Science 39:500-510.

611 Shearer BM, Cooke SB, Halenar LB, Reber SL, Plummer J, Delson E, and Tallman M. 2017. Evaluating causes of error in landmark-based data collection using scanners. PLOS One

613 12:e0187452.

614 Sholts SB, Wärmländer SKTS, Flores LM, Miller KWP, and Walker PL. 2010. Variation in the 615 measurement of cranial volume and surface area using 3D laser scanning technology. Journal of Forensic Sciences 55:871-876. 10.1111/j.1556-4029.2010.01380.x

617 Slizewski A, Friess M, and Semal P. 2010. Surface scanning of anthropological specimens: nominal-actual comparison with low cost laser scanner and high end fringe light projection surface scanning systems. Quartär 57:179-187.

Weisbecker V, and Goswami A. 2010. Brain size, life history, and metabolism at the marsupial/placental dichotomy. Proceedings of the National Academy of Sciences 107:16216-16221. 10.1073/pnas.0906486107

Williams FL, and Richtsmeier JT. 2003. Comparison of mandibular landmarks from computed tomography and 3D digitizer data. Clinical Anatomy 16:494-500. 10.1002/ca.10095

Yezerinac SM, Lougheed SC, and Handford P. 1992. Measurement error and morphometric studies - statistical power and observer experience. Systematic Biology 41:471-482. $10.2307 / 2992588$ 
628 Zelditch ML, Swiderski DL, and Sheets HD. 2012. Geometric Morphometrics for Biologists: A

629 Primer, 2nd Edition. Geometric Morphometrics for Biologists: a Primer, 2nd Edition:1$630 \quad 478$.

631 


\section{Figure 1}

Low resolution 3D surface scans compared to $\mu \mathrm{CT}$ scans of the same delicate mouse crania.

3D scans of $(A)$ dorsal view $(B)$ lateral view and $(C)$ ventral view compared to $\mu C T$ scans of

(D) dorsal view (E) lateral view and (F) ventral view. All crania are rendered in Viewbox v. 4.0.

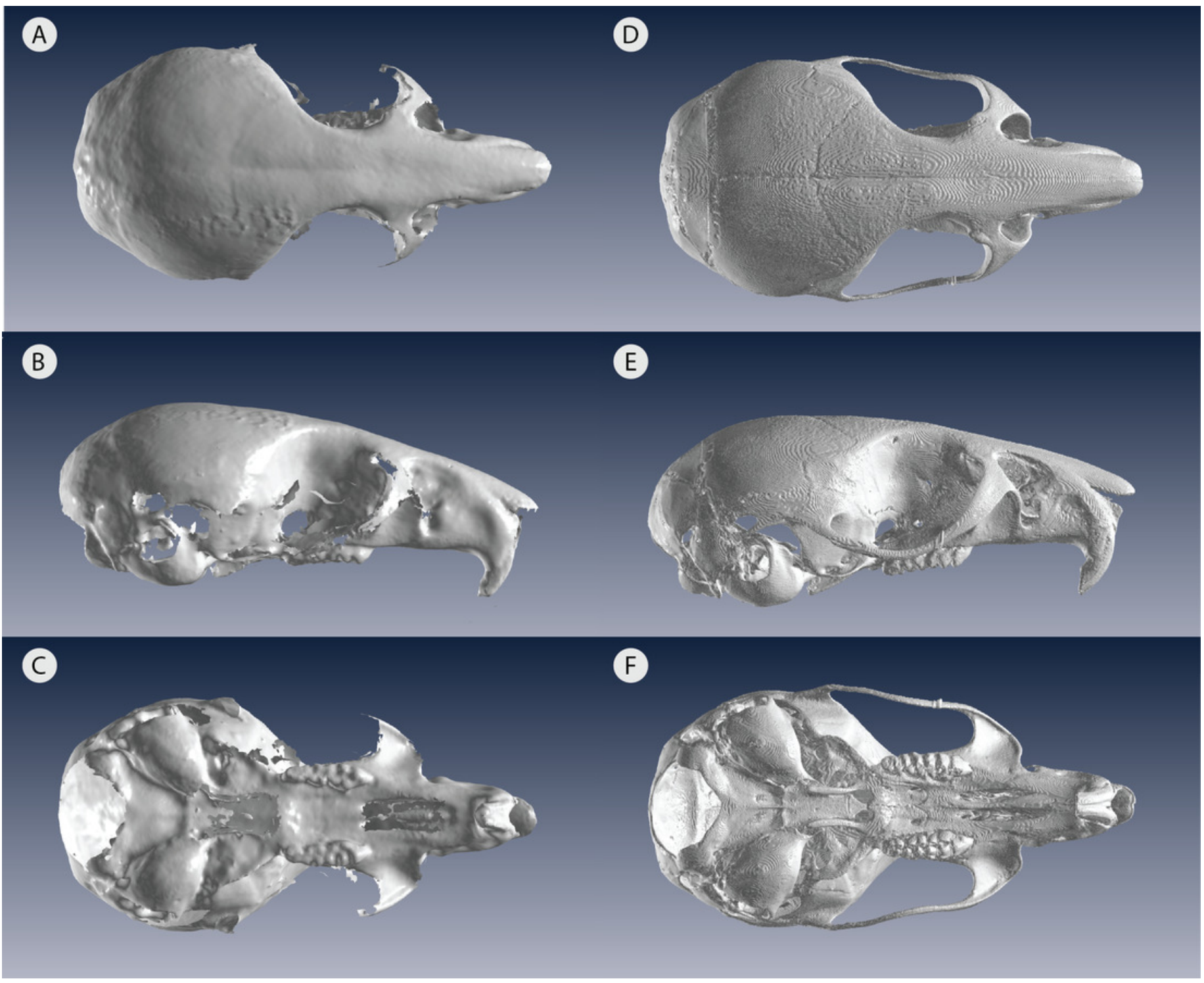




\section{Figure 2 (on next page)}

Methods flow diagram highlighting the relationship between our questions and our analyses.

(A) All delicate mouse (Pseudomys delicatulus) crania were sourced from the Queensland Museum in Brisbane, Australia. Landmarks (LMs) capture homologous points, semi-landmarks (semi-LMs) capture curves between landmarks, and patch points capture surfaces between landmarks and semi-landmarks. (B - D) These sections of questions and associated figure and table numbers summarize how we organize the paper, particularly the Results, into three sets of related analyses. 


\section{A Data Collection $(n=19)$}

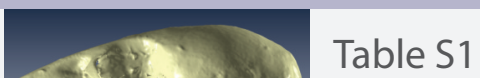

$\mu C T$ scan and 3D scan
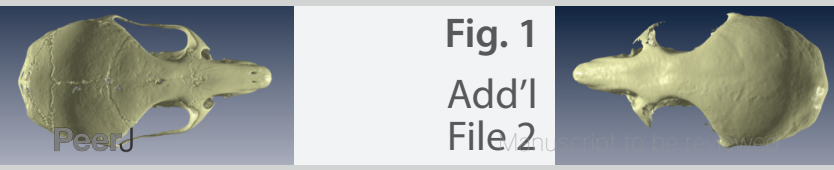

Replicate each scan x3

Landmark $(n=114)$

Fig. 3

58 LMs, 145 semi-LMs, 86 patch pts

Table S2

\section{B Analyses of shape variation}

How does variation due to scan device compare to other sources?

Table 1

... and when variation due to bilateral asymmetry is removed?

Table 2 How does variation for the symmetric shape component look?

Figs. 4 \& 5

\section{Analyses of variance and error}

Does variation among replicates differ by scan device?

Fig. 6

Does repeatability (i.e. operator error) differ by scan device?

Table 3

Does repeatability differ if fewer landmark types are used?

Table 3

\section{Analyses of intra-specific variation}

How much sexual dimorphism appears to exist in our sample?

Does one scan device provide a better basis of sex identification?
Table 4

Fig. 7

Table 5 


\section{Figure 3}

Positions of landmarks for geometric morphometric analyses.

Locations of fixed landmarks (black points), sliding semi-landmarks (red points) and sliding surface patches (purple points) on a $\mu \mathrm{CT}$ scanned individual. (A) Dorsal view of the cranium. (B) Lateral view. (C) Ventral view. Definitions are given in Table S2. 


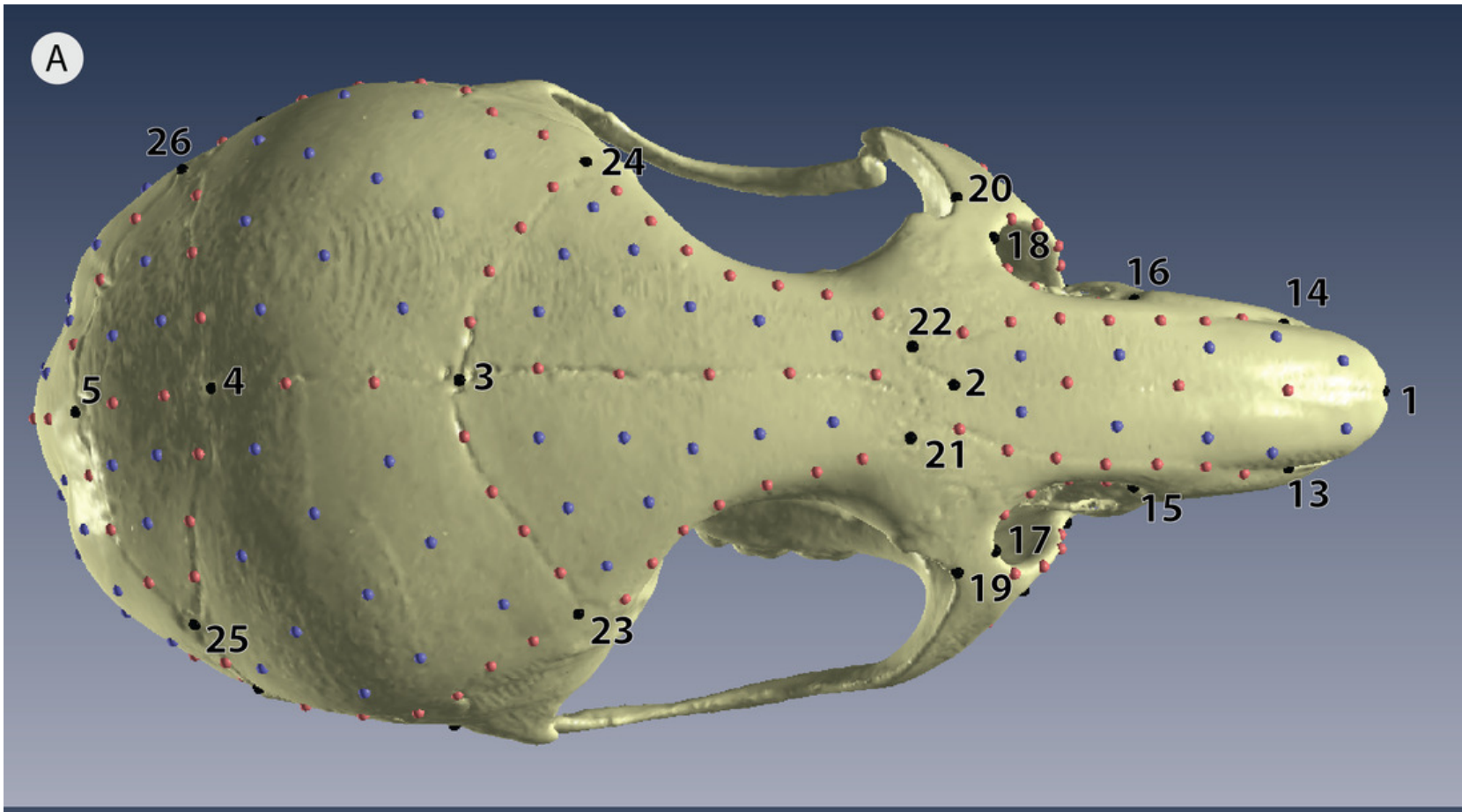

\section{B}

c
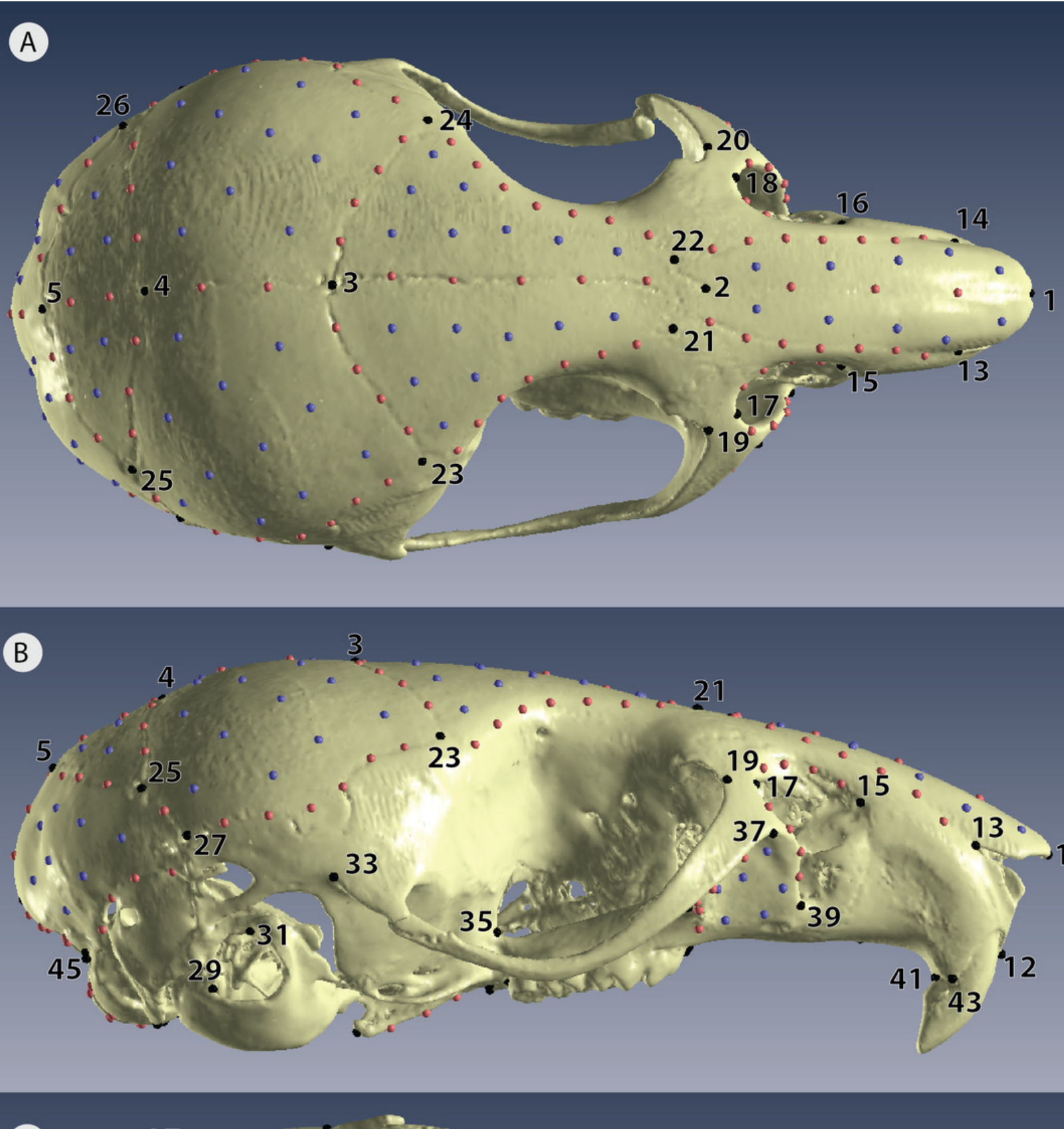


\section{Figure 4}

Exploratory PCA plots of shape variation showing differences among individuals, scan devices, and replicates of the same scan device.

(A) PC1 versus PC2 and (B) PC1 versus PC3. Each individual has a unique color shared by all of its 6 replicates. Each individual has 3 triangles to represent the 3D scanned replicates and 3 circles to represent the $\mu \mathrm{CT}$ scanned replicates. Each axis reports the total variance explained by that principal component. 


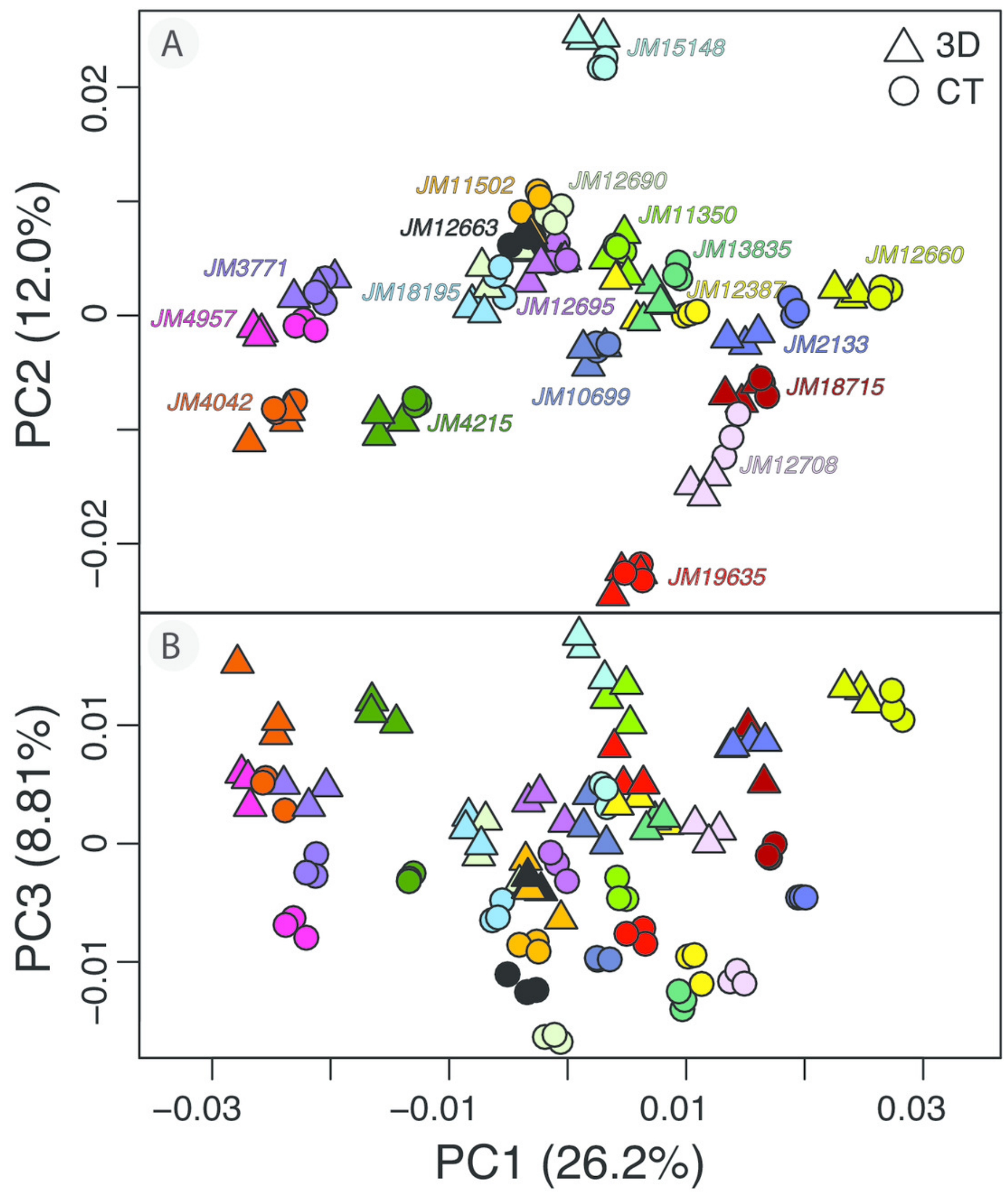




\section{Figure 5}

3D warp-grids for the three most important principal components, showing minimum and maximum shapes for each PC.

The right hand cranium shows the maximum positive shape for the principle component (PC) and the left hand cranium shows the minimum negative value. Compared to negative values (A), positive values along PC1 (26.4\% variance) correspond to a larger braincase relative to the rostrum (B). Compared to negative values (C), positive values along PC2 (11.9\% variance) correspond to a wider frontal bone compared to negative values (D). Compared to negative values $(E)$, positive values along PC3 (8.9\% variance) correspond to a more dorsally-curved ventral surface compared to negative values $(F)$.

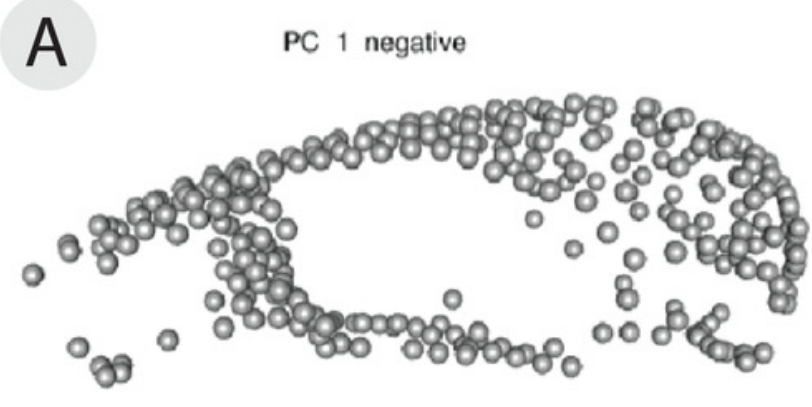

C

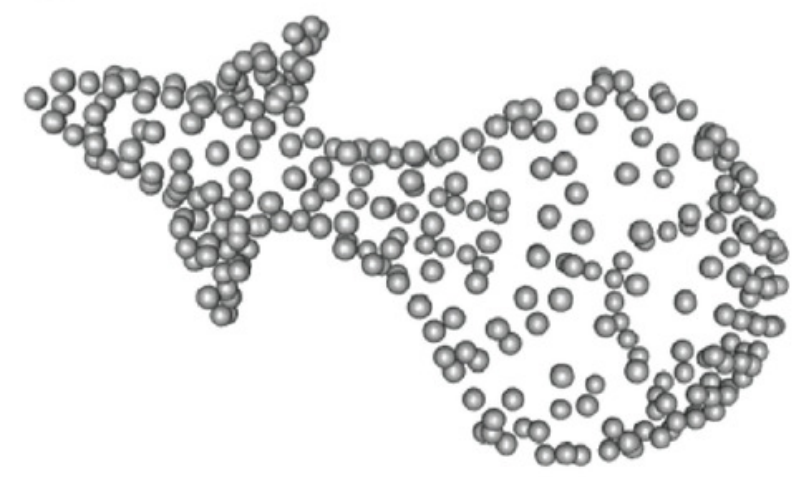

E

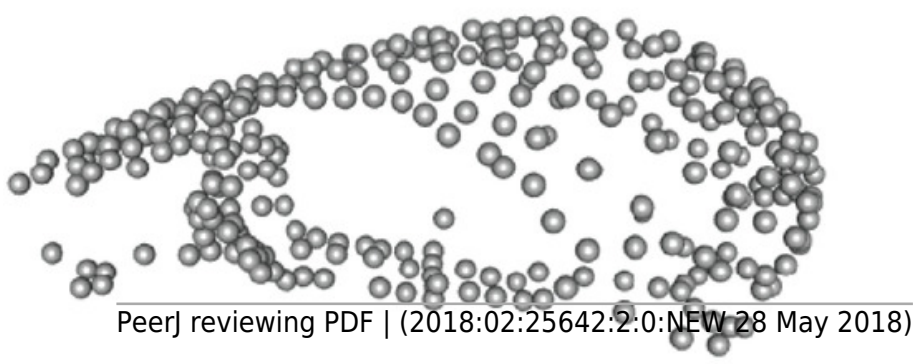

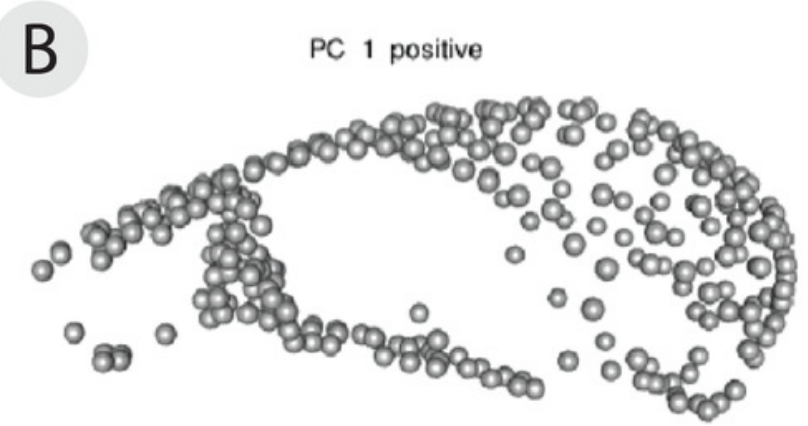

D

PC 2 positive

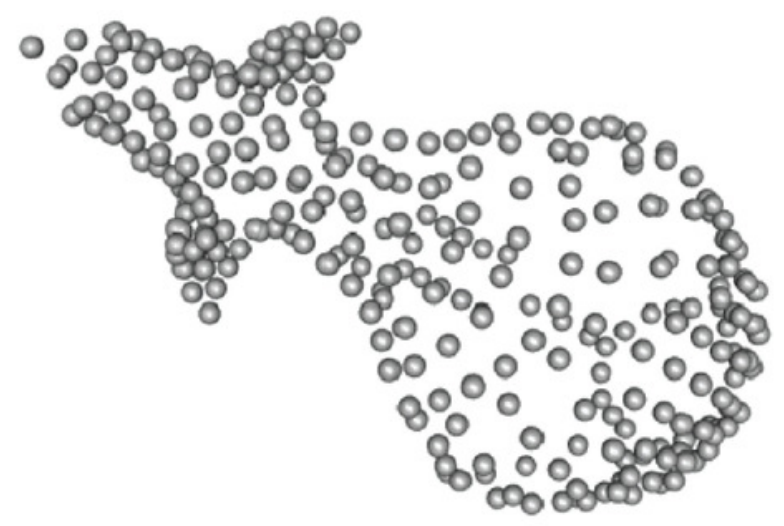

F $\quad$ PC 3 positive

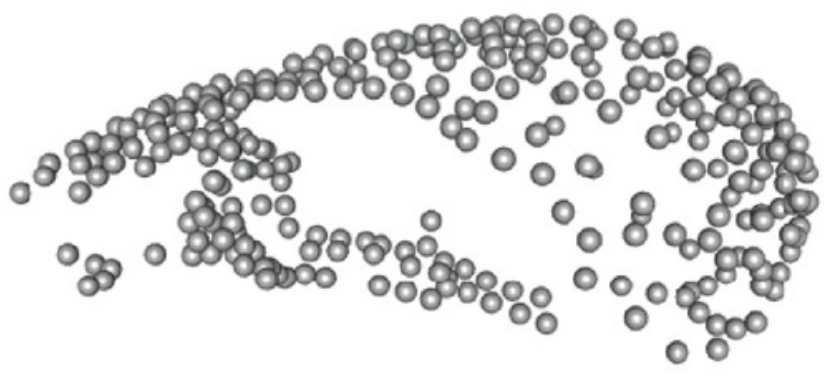


Figure 6

Morphological disparity -- as measured by shape variation among replicate scan triads -by scanning device reflects operator error.

This box plot summarizes the morphological disparity (also known as the Procrustes variance) among the three replicates of an individual for each scan type. The mean Procrustes variance for 3D scans was $1.34 \times 10^{-4}$ and $4.81 \times 10^{-5}$ for $\mu C T$ scans. This is a significant difference $(p<0.001)$.

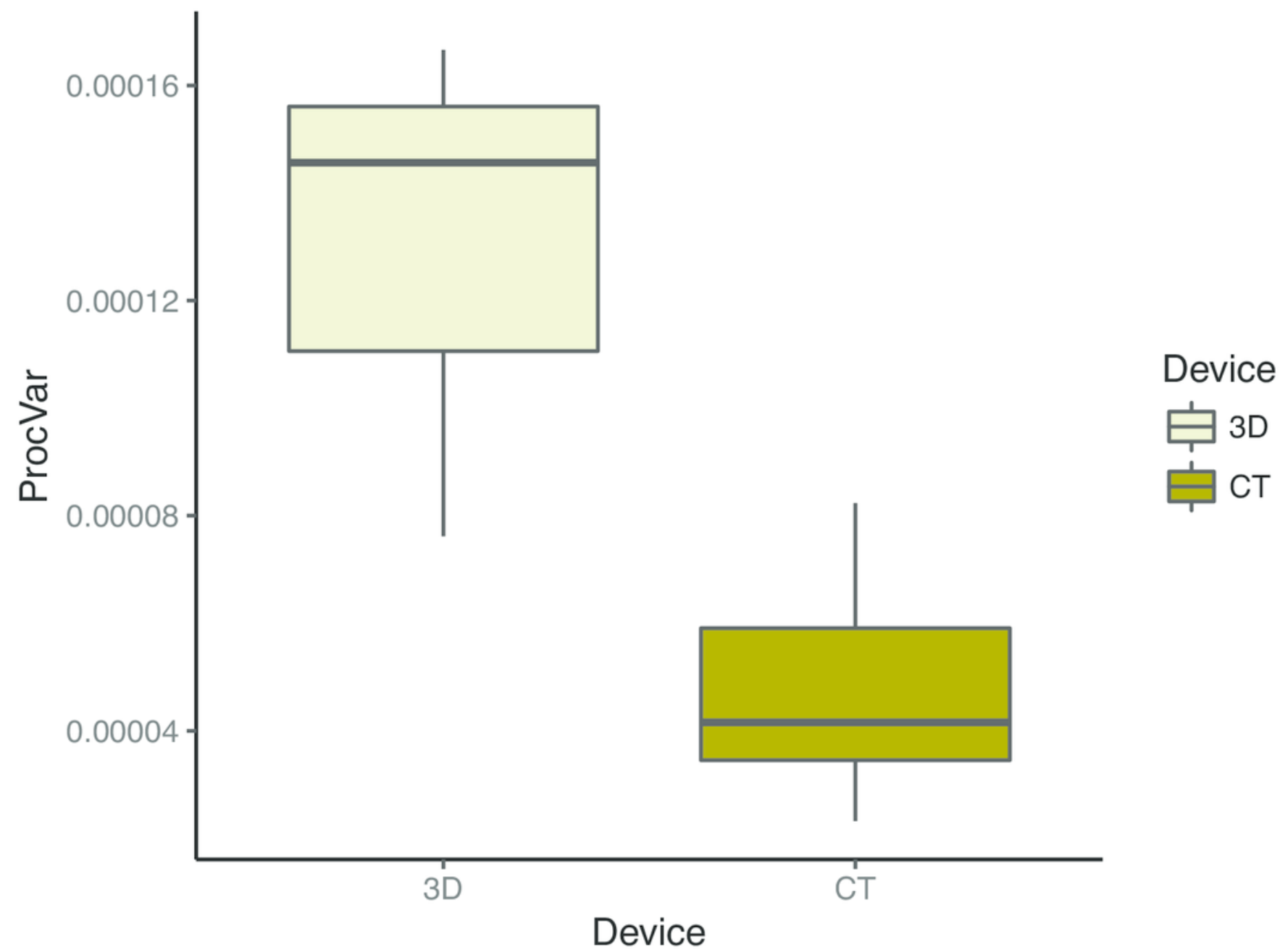




\section{Figure 7}

Intra-specific variation as shown by PCAs of 3D and $\mu \mathrm{CT}$ scan datasets colored by sex.

PCA provides an exploratory visualization of shape variation between males and females in our subsample with sex information $(n=11)$. Males $(n=4)$ are plotted in light blue and females $(n=7)$ are plotted in dark red. Results from the cross-validation test can be found in Table 5. 

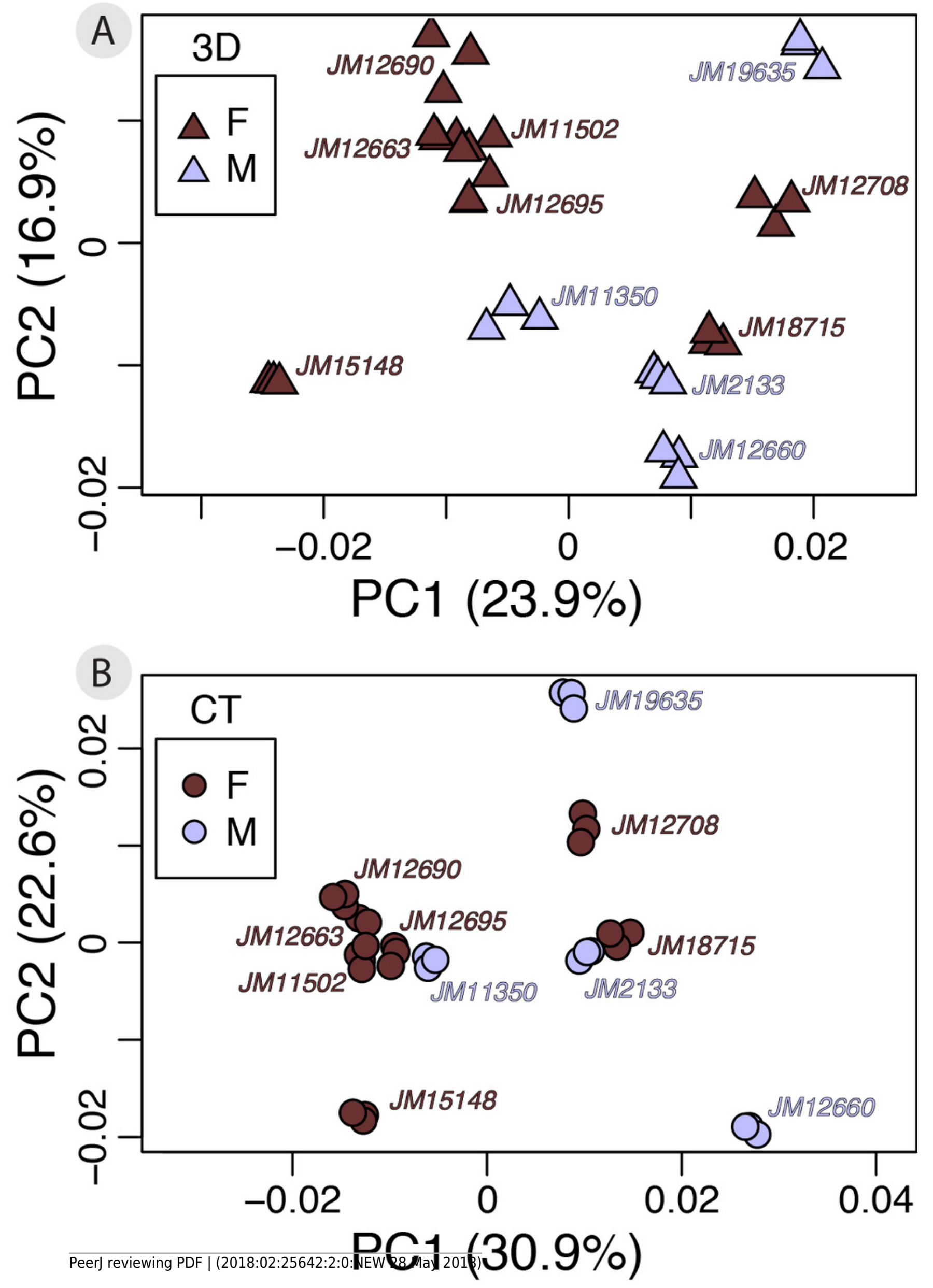


\section{Table $\mathbf{1}$ (on next page)}

General Procrustes ANOVA on sources of shape variation including asymmetry.

The \%Var column of this Procrustes ANOVA demonstrates the relative contribution of each factor to overall variation. It is calculated from the sum of squares for each factor divided by the total sum of squares for all factors. 
1

A) All Specimens

\begin{tabular}{|l|l|l|l|l|l|l|}
\hline & Df & SS & MS & \%Var & F & Pr(>F) \\
\hline Individual & 8010 & $6.21 \mathrm{E}-02$ & $7.76 \mathrm{E}-06$ & 48.3 & 11.2 & \\
& & & & & & $<.0001$ \\
\hline Side & 415 & $2.37 \mathrm{E}-02$ & $5.70 \mathrm{E}-05$ & 18.4 & 82.4 & \\
& & & & & & $<.0001$ \\
\hline Ind * Side & 7470 & $5.17 \mathrm{E}-03$ & $6.93 \mathrm{E}-07$ & 4.02 & 0.54 & 1 \\
\hline Device & 16340 & $2.08 \mathrm{E}-02$ & $1.27 \mathrm{E}-06$ & 16.1 & 4.90 & \\
& & & & & & $<.0001$ \\
\hline Res / Rep & 65360 & $1.70 \mathrm{E}-02$ & $2.59 \mathrm{E}-07$ & 13.2 & & \\
\hline
\end{tabular}

2

3 B) Only 3D Specimens

\begin{tabular}{|l|l|l|l|l|l|l|}
\hline & Df & SS & MS & \%Var & F & $\operatorname{Pr}(>$ F $)$ \\
\hline Individual & 8010 & $3.52 \mathrm{E}-02$ & $4.40 \mathrm{E}-06$ & 51.6 & 4.24 & $<.0001$ \\
\hline Side & 415 & $1.31 \mathrm{E}-02$ & $3.15 \mathrm{E}-05$ & 19.2 & 30.4 & $<.0001$ \\
\hline & & & & & & \\
Ind * Side & 7470 & $7.75 \mathrm{E}-03$ & $1.04 \mathrm{E}-06$ & 11.4 & 2.79 & $<.0001$ \\
\hline Res / Rep & 32680 & $1.22 \mathrm{E}-02$ & $3.72 \mathrm{E}-07$ & 17.8 & & \\
\hline
\end{tabular}

4

5 C) Only CT Specimens

\begin{tabular}{|l|l|l|l|l|l|l|}
\hline & Df & SS & MS & \%Var & F & $\operatorname{Pr}(>$ F $)$ \\
\hline Individual & 8010 & $3.45 \mathrm{E}-02$ & $4.31 \mathrm{E}-06$ & 61.7 & 6.41 & $<.0001$ \\
\hline Side & 415 & $1.17 \mathrm{E}-02$ & $2.81 \mathrm{E}-05$ & 20.8 & 41.8 & $<.0001$ \\
\hline Ind * Side & 7470 & $5.02 \mathrm{E}-03$ & $6.72 \mathrm{E}-07$ & 8.97 & 4.61 & $<.0001$ \\
\hline Res / Rep & 32680 & $4.76 \mathrm{E}-03$ & $1.46 \mathrm{E}-07$ & 8.52 & & \\
\hline
\end{tabular}

6 


\section{Table 2 (on next page)}

Procrustes ANOVA on the sources of shape variation using the symmetric component of shape.

The R-squared column of this Procrustes ANOVA demonstrates the relative contribution of each factor to overall variation. The shape variation of this dataset is visualized in Figures 4 and 5 . 


\begin{tabular}{|l|l|l|l|l|l|l|l|}
\hline & Df & SS & MS & Rsq & F & Z & Pr(>F) \\
\hline ind & 18 & $6.23 \mathrm{E}-02$ & $3.46 \mathrm{E}-03$ & 0.734 & 25.8 & 21.4 & 0.001 \\
\hline ind:Dev & 19 & $1.24 \mathrm{E}-02$ & $6.52 \mathrm{E}-04$ & 0.146 & 4.86 & 23.7 & 0.001 \\
\hline Residuals & 76 & $1.02 \mathrm{E}-02$ & $1.34 \mathrm{E}-04$ & 0.120 & & & \\
\hline Total & 113 & $8.49 \mathrm{E}-02$ & & & & & \\
\hline
\end{tabular}

1 


\section{Table 3 (on next page)}

Comparison of operator error in 3D scan and $\mu \mathrm{CT}$ scan datasets using Procrustes ANOVAs and repeatability scores.

The repeatability score $(R)$ is a value that reflects the ease of digitizing in a repeated measure study design. It is calculated from the Procrustes ANOVA using formulas for the intra-class correlation coefficient. The Procrustes ANOVAs were found by subsetting the dataset by scan device and by landmark types and then performing separate generalized Procrustes and bilateral symmetry alignments. (A-C) Results from the 3D-only dataset. (D-F) Results from the $\mu C T$-only dataset. $(A)$ and $(D)$ show the repeatabilites from the entire landmark datasets of each scan device. (B) and (E) remove patch points. (C) and (F) contain only fixed landmarks. 


\begin{tabular}{|l|l|l|l|l|l|l|l|l|}
\hline \multicolumn{6}{|c|}{ A) 3D Scan All Landmarks Including Patches (n = 289) } & & \\
\hline & Df & SS & MS & Rsq & F & Z & $\operatorname{Pr}(>$ F) & R \\
\hline Ind & 18 & $\begin{array}{l}3.53 \mathrm{E}- \\
02\end{array}$ & $\begin{array}{l}1.96 \mathrm{E}- \\
03\end{array}$ & 0.826 & 10.0 & 16.0 & 0.001 & $\begin{array}{l}0.75 \\
0\end{array}$ \\
\hline Residuals & 38 & $\begin{array}{l}7.46 \mathrm{E}- \\
03\end{array}$ & $\begin{array}{l}1.96 \mathrm{E}- \\
04\end{array}$ & 0.174 & & & & \\
\hline Total & 56 & $\begin{array}{l}4.28 \mathrm{E}- \\
02\end{array}$ & & & & & & \\
\hline
\end{tabular}

1

\begin{tabular}{|l|l|l|l|l|l|l|l|l|}
\hline \multicolumn{6}{|c|}{ B) 3D Scan Fixed Landmarks and Semilandmarks (n = 203) } & \\
\hline & Df & SS & MS & Rsq & F & Z & Pr(>F) & R \\
\hline Ind & 18 & $\begin{array}{l}4.37 \mathrm{E}- \\
02\end{array}$ & $\begin{array}{l}2.43 \mathrm{E}- \\
03\end{array}$ & 0.807 & 8.826 & 16.7 & 0.001 & 0.723 \\
\hline Residuals & 38 & $\begin{array}{l}1.04 \mathrm{E}- \\
02\end{array}$ & $\begin{array}{l}2.75 \mathrm{E}- \\
04\end{array}$ & 0.193 & & & & \\
\hline Total & 56 & $\begin{array}{l}5.41 \mathrm{E}- \\
02\end{array}$ & & & & & & \\
& & & & & & & \\
\hline
\end{tabular}

2

\begin{tabular}{|l|l|l|l|l|l|l|l|l|}
\hline \multicolumn{6}{|l|}{ C) 3D Scan Fixed Landmarks Only (n = 58) } & & & \\
\hline & Df & SS & MS & Rsq & F & Z & Pr(>F) & R \\
\hline Ind & 18 & $\begin{array}{l}6.90 \mathrm{E}- \\
02\end{array}$ & $\begin{array}{l}3.83 \mathrm{E}- \\
03\end{array}$ & 0.749 & 6.30 & 16.6 & 0.001 & 0.639 \\
\hline Residuals & 38 & $\begin{array}{l}2.31 \mathrm{E}- \\
02\end{array}$ & $\begin{array}{l}6.09 \mathrm{E}- \\
04\end{array}$ & 0.251 & & & & \\
\hline Total & 56 & $\begin{array}{l}9.21 \mathrm{E}- \\
02\end{array}$ & & & & & & \\
\hline
\end{tabular}

3

D) CT Scan All Landmarks Including Patches ( $\mathbf{n}=\mathbf{2 8 9}$ )

\begin{tabular}{|l|l|l|l|l|l|l|l|l|}
\hline & Df & SS & MS & Rsq & F & Z & Pr(>F) & R \\
\hline Ind & 18 & $\begin{array}{l}3.46 \mathrm{E}- \\
02\end{array}$ & $\begin{array}{l}1.92 \mathrm{E}- \\
03\end{array}$ & 0.927 & 26.9 & 18.4 & 0.001 & 0.896 \\
\hline Residuals & 38 & $\begin{array}{l}2.72 \mathrm{E}- \\
03\end{array}$ & $\begin{array}{l}7.15 \mathrm{E}- \\
05\end{array}$ & 0.073 & & & & \\
\hline Total & 56 & $\begin{array}{l}3.73 \mathrm{E}- \\
02\end{array}$ & & & & & & \\
\hline
\end{tabular}

4

\begin{tabular}{|l|l|l|l|l|l|l|l|l|}
\hline \multicolumn{6}{|c|}{ E) CT Scan Fixed Landmarks and Semilandmarks (n = 203) } & \\
\hline & Df & SS & MS & Rsq & F & Z & $\operatorname{Pr}(>$ F) & R \\
\hline Ind & 18 & $\begin{array}{l}4.33 \mathrm{E}- \\
02\end{array}$ & $\begin{array}{l}2.41 \mathrm{E}- \\
03\end{array}$ & 0.921 & 24.7 & 19.0 & 0.001 & 0.888 \\
\hline Residuals & 38 & $\begin{array}{l}3.71 \mathrm{E}- \\
03\end{array}$ & $\begin{array}{l}9.76 \mathrm{E}- \\
05\end{array}$ & 0.079 & & & & \\
& & & & & & \\
\hline
\end{tabular}




\begin{tabular}{|l|l|l|l|l|l|l|l|l|}
\hline Total & 56 & $\begin{array}{l}4.70 \mathrm{E}- \\
02\end{array}$ & & & & & & \\
\hline
\end{tabular}

5

\begin{tabular}{|c|c|c|c|c|c|c|c|c|}
\hline \multicolumn{9}{|c|}{ F) CT Scan Fixed Landmarks Only $(n=58)$} \\
\hline & Df & SS & MS & Rsq & $\mathbf{F}$ & $\mathbf{Z}$ & $\operatorname{Pr}(>F)$ & $\mathbf{R}$ \\
\hline Ind & 18 & $\begin{array}{l}6.28 \mathrm{E}- \\
02\end{array}$ & $\begin{array}{l}3.49 E- \\
03\end{array}$ & 0.893 & 17.6 & 20.2 & 0.001 & 0.847 \\
\hline $\begin{array}{l}\text { Residual } \\
\text { S }\end{array}$ & 38 & $\begin{array}{l}7.54 \mathrm{E}- \\
03\end{array}$ & $\begin{array}{l}1.98 \mathrm{E}- \\
04\end{array}$ & 0.107 & & & & \\
\hline Total & 56 & $\begin{array}{l}7.03 \mathrm{E}- \\
02\end{array}$ & & & & & & \\
\hline
\end{tabular}

6 
Table 4 (on next page)

Symmetric Procrustes ANOVA with device and sex as factors to assess relative contribution of intra-specific variation to overall shape variation.

This Procrustes ANOVA allows comparison of the relative contribution to total variation from scan device and sex (R-squared column). 


\begin{tabular}{|l|l|l|l|l|l|l|l|}
\hline & Df & SS & MS & Rsq & F & Z & Pr(>F) \\
\hline device & 1 & $2.99 \mathrm{E}-03$ & $2.99 \mathrm{E}-03$ & 0.0646 & 4.84 & 4.06 & 0.001 \\
\hline sex & 1 & $4.40 \mathrm{E}-03$ & $4.40 \mathrm{E}-03$ & 0.0952 & 7.14 & 4.96 & 0.001 \\
\hline Residuals & 63 & $3.88 \mathrm{E}-02$ & $6.16 \mathrm{E}-04$ & & & & \\
\hline Total & 65 & $4.62 \mathrm{E}-02$ & & & & & \\
\hline
\end{tabular}

1 


\section{Table 5 (on next page)}

Between group PCA classification test to assess whether one scan device dataset performs better at identifying sexes based on shape.

This analysis averages shape among replicates, computes a between-group PCA separately for $3 \mathrm{D}$ and $\mu \mathrm{CT}$ datasets, and runs a cross-validation classification test. The results indicate whether one type of scan dataset is more successful at classifying males versus females based on the shape variation present in the dataset. It also returns a kappa statistic; a kappa value over 0.20 indicates "fair" agreement between the two datasets. Shape variation visualized by sex can be seen in Figure 7 . 


\begin{tabular}{|c|c|c|}
\hline \multicolumn{3}{|c|}{ Cross-validated classification results in frequencies } \\
\hline 3D & f & m \\
\hline$f(n=7)$ & 5 & 2 \\
\hline$m(n=4)$ & 2 & 2 \\
\hline CT & $f$ & $\mathbf{m}$ \\
\hline$f(n=7)$ & 5 & 2 \\
\hline$m(n=4)$ & 2 & 2 \\
\hline \multicolumn{3}{|c|}{ Cross-validated classification results in \% } \\
\hline 3D & f & m \\
\hline f & 71.4 & 28.6 \\
\hline $\mathbf{m}$ & 50.0 & 50.0 \\
\hline $\mathbf{C T}$ & $f$ & m \\
\hline$f$ & 71.4 & 28.6 \\
\hline m & 50.0 & 50.0 \\
\hline \multicolumn{3}{|c|}{ Overall classification accuracy (\%) } \\
\hline 3D & 63.6 & \\
\hline CT & 63.6 & \\
\hline \multicolumn{3}{|c|}{ Kappa statistic } \\
\hline 3D & 0.214 & \\
\hline CT & 0.214 & \\
\hline
\end{tabular}

1 